\title{
Vasoprotective Functions of High-Density Lipoproteins Relevant to Alzheimer's Disease Are Partially Conserved in Apolipoprotein B-Depleted Plasma
}

\author{
Emily B. Button 1,2 (D), Megan Gilmour ${ }^{1,2}$, Harleen K. Cheema ${ }^{1,2}$, Emma M. Martin ${ }^{1,2}$, \\ Andrew Agbay 1,2, Jérôme Robert ${ }^{1,2,+}$ and Cheryl L. Wellington 1,2, $*,+$ \\ 1 Department of Pathology and Laboratory Medicine, University of British Columbia, Vancouver, \\ BC V6T 1Z3, Canada; ebbutton@mail.ubc.ca (E.B.B.); meg.gilmour@gmail.com (M.G.); \\ harleencheema@hotmail.com (H.K.C.); emmarie@mail.ubc.ca (E.M.M.); \\ andrew.agbay@ubc.ca (A.A.); jerome.robert@ubc.ca (J.R.) \\ 2 Djavad Mowafaghian Centre for Brain Health, Department of Pathology and Laboratory Medicine, \\ University of British Columbia, Vancouver, BC V6T 1Z3, Canada \\ * Correspondence: cheryl.wellington@ubc.ca; Tel.: +1-604-827-3769 \\ + Co-senior authors.
}

Received: 7 January 2019; Accepted: 21 January 2019; Published: 22 January 2019

\begin{abstract}
High-density lipoproteins (HDL) are known to have vasoprotective functions in peripheral arteries and many of these functions extend to brain-derived endothelial cells. Importantly, several novel brain-relevant HDL functions have been discovered using brain endothelial cells and in 3D bioengineered human arteries. The cerebrovascular benefits of HDL in healthy humans may partly explain epidemiological evidence suggesting a protective association of circulating HDL levels against Alzheimer's Disease (AD) risk. As several methods exist to prepare HDL from plasma, here we compared cerebrovascular functions relevant to AD using HDL isolated by density gradient ultracentrifugation relative to apoB-depleted plasma prepared by polyethylene-glycol precipitation, a common high-throughput method to evaluate HDL cholesterol efflux capacity in clinical biospecimens. We found that apoB-depleted plasma was functionally equivalent to HDL isolated by ultracentrifugation in terms of its ability to reduce vascular $A \beta$ accumulation, suppress $\mathrm{TNF} \alpha$-induced vascular inflammation and delay A $\beta$ fibrillization. However, only HDL isolated by ultracentrifugation was able to suppress $A \beta$-induced vascular inflammation, improve $A \beta$ clearance, and induce endothelial nitric oxide production.
\end{abstract}

Keywords: high-density lipoprotein (HDL); cerebrovasculature; endothelial cell; amyloid beta (A $\beta$ ); Alzheimer's disease; blood-brain barrier (BBB); inflammation; cerebral amyloid angiopathy (CAA)

\section{Introduction}

An emerging area of interest for high-density lipoprotein (HDL) research is its role in neurodegenerative disease. Alzheimer's disease (AD) is the principal form of dementia and has a tremendous social and economic burden worldwide [1]. Cardiovascular disease (CVD) risk factors including hypertension, type II diabetes mellitus (T2DM), and hypercholesterolemia augment AD risk. Furthermore, cerebrovascular dysfunction, including vascular accumulation of amyloid beta $(\mathrm{A} \beta)$ known as cerebral amyloid angiopathy (CAA), is found in the majority of AD cases [2,3]. Therefore, a better understanding of the vascular contributions to cognitive impairment and dementia is a priority research area [4]. The inverse association of plasma apoA-I and HDL-cholesterol (HDL-C) levels 
with AD risk in some [5,6], but not all [7-9] epidemiological studies suggests that HDL may have functional properties that protect against $\mathrm{AD}$ that are not necessarily captured by measurement of apoA-I or HDL-C levels per se. Mechanistically, preclinical studies show that HDL-deficient AD mice, generated by genetic deletion of apoA-I, have increased vascular $\mathrm{A} \beta$ deposition, known as CAA, and worse cognitive performance compared to AD mice with normal HDL levels [10], whereas AD mice overexpressing apoA-I from its native promoter show the opposite phenotype with an additional improvement in central nervous system (CNS) inflammation [11]. Furthermore, oral treatment of $\mathrm{AD}$ mice with an apoA-I mimetic improves memory and reduces amyloid burden [12], intravenous treatment with reconstituted human HDL or recombinant apoA-I Milano reduces soluble A $\beta$ levels in the brains of AD mice [13,14], and either treatment reduces CNS inflammation [12,14].

Our group recently described several cerebral vasoprotective functions of HDL relevant to AD using a blood-brain barrier (BBB) cell line (hCMEC/d3) and a novel 3-dimensional (3D) perfusible cerebrovascular in vitro model composed of primary human endothelial cells (EC) and smooth muscle cells (SMC). We reported that HDL from young, healthy human donors has at least four vasoprotective functions on brain vessels that are relevant to $A D$, including: (i) reducing $A \beta$ vascular accumulation and increasing transport through the vasculature; (ii) inhibiting $\mathrm{A} \beta$-induced endothelial activation; (iii) delaying $A \beta$ fibrillization and (iv) inducing nitric oxide (NO) production $[15,16]$.

It is now of interest to assess these novel AD-relevant HDL functions in clinical biospecimens, which are typically available in smaller volumes than the $\sim 3 \mathrm{~mL}$ of plasma or serum required for traditional ultracentrifugation purification of HDL. Clinical assays of cholesterol efflux capacity, the best-established HDL functional assay, use plasma treated with polyethylene glycol (PEG) to deplete non-HDL lipoproteins [17-19]. While apoB-depleted plasma is not "HDL" as many other plasma components remain in the preparation, it is a more cost-effective and higher-throughput method than ultracentrifugation and requires much less starting material. Consequently, the benefits and weaknesses of apoB-depleted plasma must be weighed against those of HDL isolated by ultracentrifugation when developing novel assays of HDL function. Therefore, this study was designed to evaluate how the pre-analytic factors of ultracentrifugation and PEG precipitation affect assay performance of cerebrovascular functions of HDL that are relevant to $A D$, namely increasing $A \beta$ vascular clearance, anti-inflammatory activity against $A \beta$, delay of $A \beta$ fibrillization and promotion of NO secretion.

\section{Results}

\subsection{ApoB-Depleted Plasma Reduces A $\beta$ Accumulation in Bioengineered Vessels Similar to High-Density} Lipoprotein (HDL) Isolated by Ultracentrifugation But Does Not Effectively Increase A $\beta$ Transport across the Vessel Wall

We developed an in vitro, 3D model of CAA using primary human EC and SMC cells cultured under native-like flow conditions (Figure S1a-c) and showed that when HDL isolated by ultracentrifugation is circulated through the lumen of the arteries, the accumulation of $A \beta 42$ in the arterial wall is prevented [15]. In the present study, we used this 3D human CAA model to assess whether apoB-depleted plasma produced by PEG-precipitation reproduces the protective effects of purified HDL related to A $\beta 42$ deposition and transport. After normalizing to cholesterol concentrations, HDL and apoB-depleted plasma were circulated at $10.5 \mathrm{mg} / \mathrm{dL}$ through bioengineered arteries. This dose mirrors that previously used [15,16] and corresponds to $20 \%$ and $25 \%$ of normal plasma HDL-C concentrations for healthy adult females and males, respectively [20]. We observed that HDL isolated by ultracentrifugation and apoB-depleted plasma were equally effective in reducing soluble A 342 levels in vascular tissue after $24 \mathrm{~h}$; from $61.17 \mathrm{ng} / \mathrm{mg}$ to $17.49 \mathrm{ng} / \mathrm{mg}(p=0.009)$ and $27.69 \mathrm{ng} / \mathrm{mg}(p=0.036)$ for HDL and apoB-depleted plasma, respectively (Figure 1a), albeit with higher variability observed with apoB-depleted plasma. We also measured A $\beta 42$ transported through the vessel wall into the circulation over the first $4 \mathrm{~h}$ of treatment and also at $24 \mathrm{~h}$. Over the first $4 \mathrm{~h}$ (Figure $1 \mathrm{~b}$ ) and after $24 \mathrm{~h}$ (Figure $1 \mathrm{c}$ ), only HDL significantly promoted A $\beta 42$ transport into the 
circulation compared to A $\beta 42$ alone ( $p=0.020, p=0.005$ respectively). More specifically, HDL treatment increased the amount of $A \beta 42$ transported into the circulation from $21.75 \mathrm{ng} / \mathrm{mL}$ to $25.67 \mathrm{ng} / \mathrm{mL}$ whereas $A \beta 42$ in the circulating media after treatment with apoB-depleted plasma only reached $22.15 \mathrm{ng} / \mathrm{mL}$. A $\beta 42$ transport was also significantly different between tissues treated with HDL and those treated with apoB-depleted plasma over the $4 \mathrm{~h}$ and after $24 \mathrm{~h}(p=0.0006, p=0.010$ respectively). Importantly, A $\beta 42$ levels in ultracentrifuge-isolated HDL and apoB-depleted plasma were below the detection limit as assessed by enzyme-linked immunosorbent assay (ELISA), therefore, the differences in $A \beta 42$ measured in the circulation media cannot be attributed to $A \beta 42$ bound to HDL. However, it is possible that non-HDL plasma components, such as albumin or immunoglobulins, or residual PEG solution present in the apoB-depleted plasma, could mask the A $\beta 42$ epitope used by the ELISA. Therefore, the lack of observed effect of apoB-depleted plasma on A $\beta 42$ transport through the vessel wall may be due to a technical limitation.

a

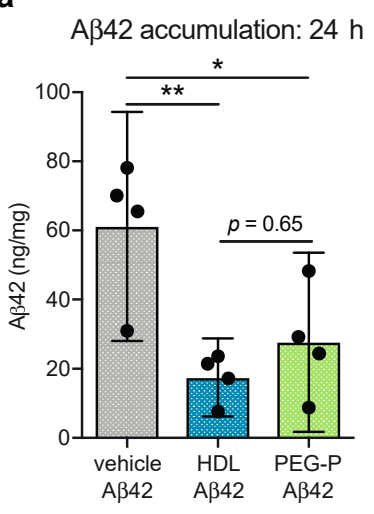

b

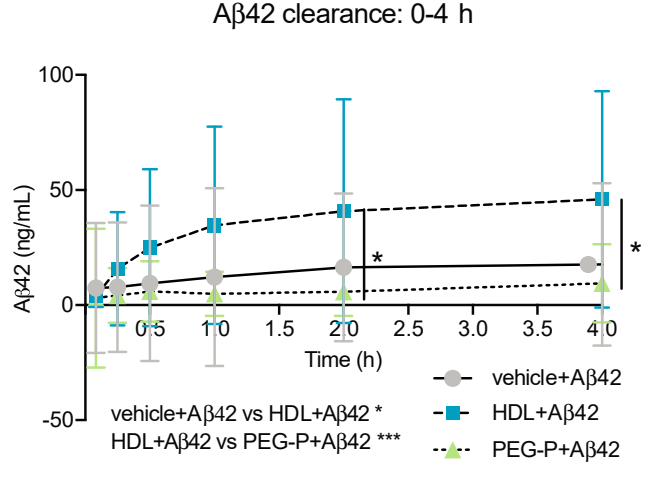

c

A $\beta 42$ clearance: $24 \mathrm{~h}$

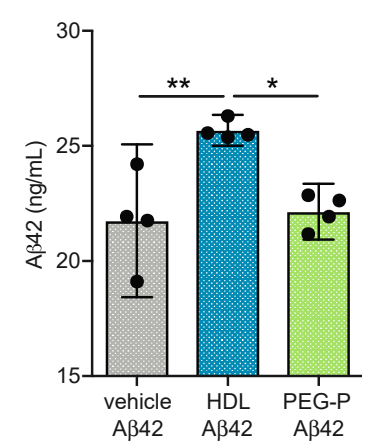

Figure 1. ApoB-depleted plasma reduces $A \beta$ accumulation in bioengineered vessel similar to HDL isolated by ultracentrifugation but does not increase $A \beta$ transport across the vessel wall in the same manner. $3 \mathrm{D}$ bioengineered human vessels were subjected to abluminal $\mathrm{A} \beta 42$ treatment $(1 \mu \mathrm{mol} / \mathrm{L})$ with or without luminal HDL ( $200 \mu \mathrm{g} / \mathrm{mL}$ protein, $10.5 \mathrm{mg} / \mathrm{dL}$ HDL-C) or apoB-depleted plasma $(10.5 \mathrm{mg} / \mathrm{dL}$ HDL-C) treatment. A $\beta 42$ level were measured in (a) tissue homogenates after $24 \mathrm{~h}$ and in circulating media (b) over $4 \mathrm{~h}$ and (c) after $24 \mathrm{~h}$ by enzyme-linked immunosorbent assay (ELISA). Scatter plots represent independent experiments with mean $\pm 95 \%$ confidence interval. ${ }^{*} p<0.05,{ }^{* *} p<0.01$, or exact $p$-value by one-way analysis of variance (ANOVA) with Tukey's multiple comparisons test for (a) and (c). ${ }^{*} p<0.05,{ }^{* * *} p<0.001$ omnibus analysis by two-way ANOVA displayed below the graph, ${ }^{*} p<0.05$ by Sidak's multiple comparisons test displayed within the graph for (b). A $\beta 42$ : amyloid beta 42; HDL: high-density lipoproteins isolated by sequential density gradient ultracentrifugation; PEG-P: apoB-depleted plasma by polyethylene glycol precipitation.

2.2. ApoB-Depleted Plasma Does Not Retain the Ability of HDL to Reduce A $\beta$-Induced Monocyte Binding to the Endothelium

$\mathrm{AD}$ is associated with cerebrovascular inflammation and $\mathrm{A} \beta$ has been reported by several independent groups to activate EC [21-23]. Previously, we demonstrated that HDL isolated by ultracentrifugation from the plasma of young healthy donors reduced $A \beta$-induced monocyte binding to EC [16]. To asses this anti-inflammatory function of HDL in apoB-depleted plasma, we performed monocyte-binding assays where HDL or apoB-depleted plasma were circulated through $3 \mathrm{D}$ bioengineered arteries that were treated abluminally with $\mathrm{A} \beta 42$ for $21 \mathrm{~h}$ after which fluorescently labelled monocytes were added to the circulation for $3 \mathrm{~h}$. Quantification of adhered monocytes to the vessel lumen demonstrated that circulating HDL reduced endothelial activation from a mean of 16.57 adhered monocytes to 5.68 cells but that circulation of apoB-depleted plasma did not significantly affect monocyte adhesion with a mean of 12.64 adhered cells observed ( $p=0.011$ HDL vs. vehicle, $p=0.435$ apoB-depleted plasma vs. vehicle, $p=0.101 \mathrm{HDL}$ vs. apoB-depleted plasma) (Figure 2a). 
Recently we reported that HDL suppresses A $\beta$-induced peripheral blood mononuclear cell (PBMC) binding to brain-derived EC in a mechanism distinct from TNF $\alpha$-induced PBMC adhesion [16]. To further investigate whether the lack of response observed with apoB-depleted plasma is unique to $\mathrm{A} \beta$ or extends to all inflammatory stimuli, we measured PBMC adhesion to the well-characterized BBB cell model hCMEC/d3 cultured in regular tissue culture plates. First, we confirmed that apoB depleted plasma did not suppress $A \beta 42$-induced endothelial activation in this $2 \mathrm{D}$ model. We observed that pretreatment of $\mathrm{hCMEC} / \mathrm{d} 3$ for $2 \mathrm{~h}$ with HDL isolated by ultracentrifugation reduced the mean number of adhered PBMC to A $\beta 42$ stimulated endothelial cells from 8.6 to 3.04 PBMC while pretreatment with apoB-depleted plasma did not significantly change PBMC adhesion $(p=0.037$ vehicle vs. $A \beta 42$ alone, $p=0.006 \mathrm{~A} \beta 42$ alone vs. $\mathrm{A} \beta 42$ with HDL, $p=0.794 \mathrm{~A} \beta 42$ alone vs. $\mathrm{A} \beta 42$ with apoB-depleted plasma, $p=0.050 \mathrm{~A} \beta 42$ with HDL vs. A $\beta 42$ with apoB-depleted plasma) (Figure $2 b$ ). On the other hand, both preparations significantly suppressed PBMC adhesion in hCMEC/d3 stimulated with the classical inflammatory stimulus tumor necrosis factor alpha (TNF $\alpha$ ) from a mean of 14.39 to 6.65 and 7.40 adhered PBMC for HDL and apoB-depleted plasma, respectively $(p=0.001$ vehicle vs. TNF $\alpha, p=0.007 \mathrm{TNF} \alpha$ alone vs. TNF $\alpha$ with HDL, and $p=0.017 \mathrm{TNF} \alpha$ alone vs. TNF $\alpha$ with apoB-depleted plasma) (Figure 2c).

a

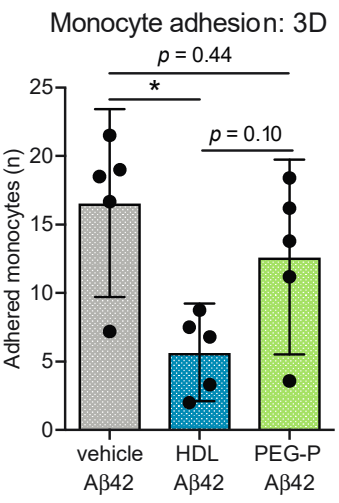

b

PBMC adhesion: 2D

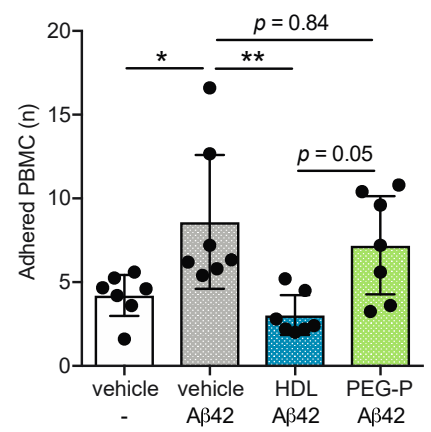

C

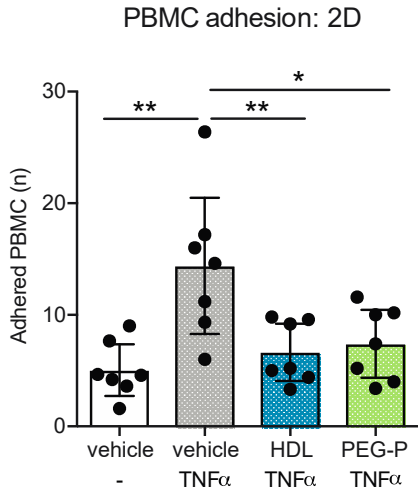

Figure 2. ApoB-depleted plasma does not maintain the ability of HDL to reduce A $\beta$-induced monocyte binding to the endothelium. 3D bioengineered human vessels were treated with abluminal $\mathrm{A} \beta 42$ $(1 \mu \mathrm{mol} / \mathrm{L})$ and luminal HDL $(200 \mu \mathrm{g} / \mathrm{mL}$ protein, $10.5 \mathrm{mg} / \mathrm{dL}$ HDL-C) or apoB-depleted plasma (10.5 mg/dL HDL-C) for $21 \mathrm{~h}$ before fluorescently labelled THP-1 monocytes were added to the circulating media for $3 \mathrm{~h}$. (a) The monocytes adhered to the lumen of the bioengineered vessels were visualized with fluorescently microscopy and counted. Brian-derived endothelial cells (hCMEC/d3) in monoculture were pretreated with HDL (100 $\mu \mathrm{g} / \mathrm{mL}$ protein, $5 \mathrm{mg} / \mathrm{dL}$ HDL-C) or apoB-depleted plasma ( $5 \mathrm{mg} / \mathrm{dL}$ HDL-C) for $2 \mathrm{~h}$ then stimulated for $3 \mathrm{~h}$ with (b) A $\beta 42(0.1 \mu \mathrm{mol} / \mathrm{L})$ or (c) TNF $\alpha$ $(1 \mathrm{ng} / \mathrm{mL}$ ) before the addition of fluorescently labelled PBMC for $3 \mathrm{~h}$. The PBMC adhered to the endothelial cells were visualized with fluorescence microscopy and counted. Scatter plots represent independent experiments with mean $\pm 95 \%$ confidence interval. ${ }^{*} p<0.05,{ }^{* *} p<0.01$, or exact $p$-value by one-way ANOVA with Tukey's multiple comparisons test. HDL: high-density lipoproteins isolated by sequential density gradient ultracentrifugation; PEG-P: apoB-depleted plasma by polyethylene glycol precipitation; TNF $\alpha$ : tumor necrosis factor alpha; PBMC: peripheral blood mononuclear cell; A $\beta 42$ : amyloid beta 42 .

\subsection{ApoB-Depleted Plasma Is Functionally Equivalent to HDL with Respect to Delaying A 42 Fibrillization}

We have previously shown that HDL isolated by ultracentrifugation from healthy human donors can delay A $\beta 42$ fibrillization in a cell-free Thioflavin $T$ assay [16]. Therefore, we used this assay to compare HDL isolated by ultracentrifugation against apoB-depleted plasma produced by PEG-precipitation and found that both preparations delayed the onset of $A \beta 42$ fibrillization to an equivalent extent (Figure 3a). Fluorescence curves were fitted to a Boltzman sigmoidal curve 
to determine the time to half-maximal fluorescence $\left(t_{50}\right)$ and maximal fluorescence. The $t_{50}$ was significantly delayed with the addition of either HDL or apoB-depleted plasma from $3.85 \mathrm{~h}$ to $9.22 \mathrm{~h}$ and $7.84 \mathrm{~h}$, respectively $(p=0.001, p=0.004)$ (Figure $3 \mathrm{~b}$ ). However, we note that the curve-fitting approach used here may require future optimization due to the distinct shape of the apoB-depleted plasma curve. Although the data for apoB-depleted plasma with $\mathrm{A} \beta 42$ fit the Boltzman sigmoidal curve well $\left(R^{2}=0.94\right)$, the sharp increase in fibrillization observed with vehicle and HDL is not evident with apoB-depleted plasma, and the maximal fluorescence observed in samples containing HDL isolated by ultracentrifugation is significantly higher compared to $\mathrm{A} \beta 42$ alone and apoB-depleted plasma (50,895 relative fluorescence units (RFU) vs. 30,090 RFU and 29,324 RFU respectively, $p=0.044$ and $p=0.038$ ) (Figure 3c).

a

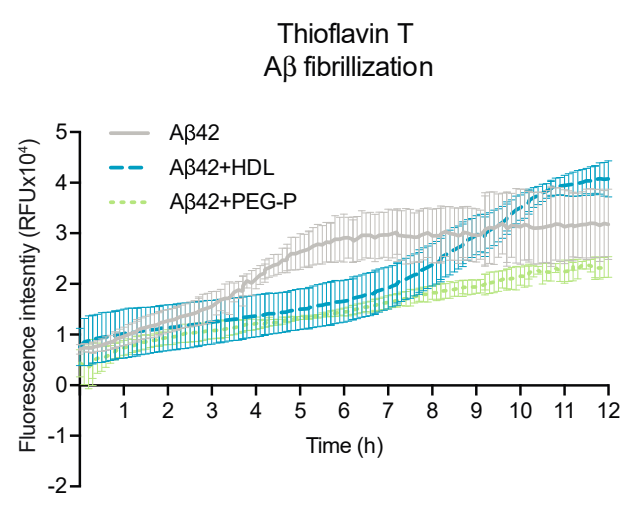

b

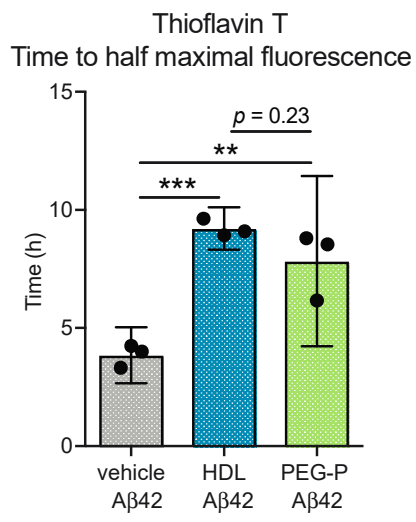

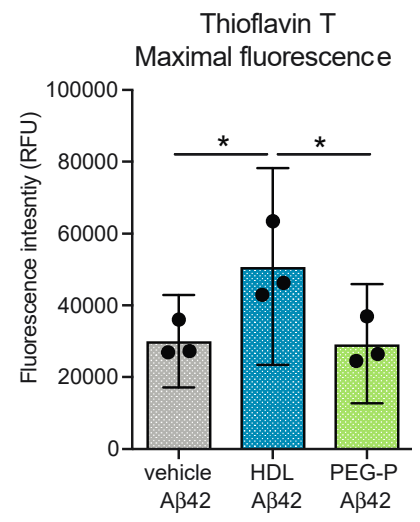

Figure 3. ApoB-depleted plasma is sufficient to assay the anti-fibrillary effects of HDL against A $\beta 42$. (a) The formation of $\beta$-amyloid pleated sheets was measured in a cell-free assay using thioflavin T over $12 \mathrm{~h}$. Data were fit to a Boltzman curve to determine the (b) time to half-maximal fluorescence and (c) maximal fluorescence. Scatter plots represent independent experiments with mean $\pm 95 \%$ confidence interval. Time course data is represented with a point for mean \pm standard deviation (SD). ${ }^{*} p<0.05$, ${ }^{* *} p<0.01,{ }^{* * *} p<0.001$, or exact $p$-value by one-way ANOVA with Tukey's multiple comparisons test. RFU: relative fluorescence units; HDL: high-density lipoproteins isolated by sequential density gradient ultracentrifugation; PEG-P: apoB-depleted plasma by polyethylene glycol precipitation.

\subsection{ApoB-Depleted Plasma Does Not Induce Nitric Oxide (NO) in Human Brain-Derived Endothelial Cells}

A key vascular protective function of HDL is the ability to induce NO production in EC via the phosphorylation of endothelial nitric oxide synthase [24]. We have previously shown that HDL isolated by ultracentrifugation maintains this function in brain-microvascular EC and that the mechanism by which HDL induces NO production in these cells is distinct from other anti-inflammatory mechanisms [16]. Here we compared the ability of HDL isolated by ultracentrifugation and apoB-depleted plasma to induce NO production in hCMEC/d3. Treatment with HDL for $4 \mathrm{~h}$ increased intracellular NO levels from a log fluorescence intensity of $2.14 \mathrm{RFU}$ to $2.57 \mathrm{RFU}$ ( $p=0.028)$, however, the intracellular levels of $\mathrm{NO}$ in cells treated with apoB-depleted plasma were unchanged $(p=0.994$ vehicle vs. apoB-depleted plasma, $p=0.027 \mathrm{HDL}$ vs. apoB-depleted plasma) (Figure 4). 
Nitric oxide production

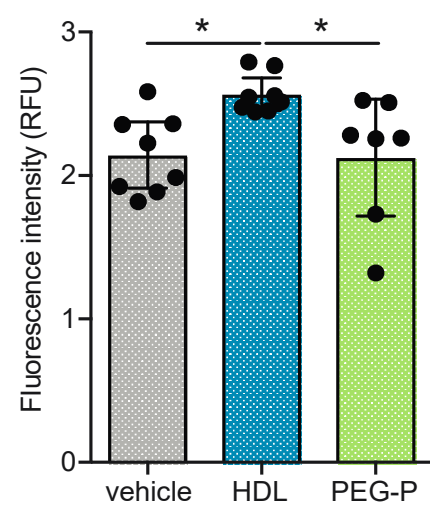

Figure 4. ApoB-depleted plasma does not recapitulate the nitric oxide- (NO) inducing abilities of isolated by ultracentrifugation in human brain-derived endothelial cells. NO production in hCMEC/d3 was measured using the fluorescent indicator DAF-2 DA and HDL treatment $(100 \mu \mathrm{g} / \mathrm{mL}$ protein, $5 \mathrm{mg} / \mathrm{dL}$ HDL-C) for $4 \mathrm{~h}$. Scatter plots represent independent experiments with mean $\pm 95 \%$ confidence interval. ${ }^{*} p<0.05$ by one-way ANOVA with Tukey's multiple comparisons test. RFU: relative fluorescence units; HDL: high-density lipoproteins isolated by sequential density gradient ultracentrifugation; PEG-P: apoB-depleted plasma by polyethylene glycol precipitation; DAF-2 DA: 4,5-diaminofluorescein diacetate.

2.5. Non-HDL Plasma Components in ApoB-Depleted Plasma Interfere with Some Anti-Inflammatory Activities of Purified HDL

Two hypotheses were tested to explain why apoB-depleted plasma fails to reduce $A \beta$-induced monocyte binding and NO production. First, residual PEG polymer can remain in apoB-depleted plasma even after extensive dialysis, which may interfere with specific HDL functions due to a reduction in the hydration shell surrounding the HDL particles [25]; second, apoB-depleted plasma contains abundant non-HDL plasma proteins, which may also interfere with certain HDL functions. Two additional specimen processing protocols were used to test these hypotheses. First, we tested whether PEG interferes with HDL functions by first using PEG-precipitation to deplete apoB-containing lipoproteins followed by ultracentrifugation to remove non-HDL plasma proteins, thereby producing HDL that has been exposed to PEG polymers (Figure S2c). To test whether non-HDL plasma components interfere with specific HDL activities we used a single ultracentrifugation step to produce an isolate with a similar composition to PEG-precipitated plasma but with no exposure to PEG (Figure S2d).

HDL isolated by ultracentrifugation and HDL isolated by the combination of PEG-precipitation and ultracentrifugation suppressed $\mathrm{A} \beta$-induced PBMC adhesion from 5.48 average cell counts to $1.93(p=0.003)$ and 2.89 average cell counts $(p=0.037)$, respectively, whereas neither preparation of apoB-depleted plasma had any significant effect on PBMC adhesion (Figure 5a). Similarly, HDL isolated by ultracentrifugation and by the combination method increased NO-production from a $\log$ fluorescence intensity of $3.64 \mathrm{RFU}$ to $4.92 \mathrm{RFU}(p=0.047)$ and $4.15 \mathrm{RFU}(p=0.006)$, respectively, while neither preparation of apoB-depleted plasma significantly altered NO production (Figure $5 b$ ). These observations support the hypothesis that non-HDL plasma components present in apoB-depleted plasma, rather than exposure of HDL particles to PEG, are responsible for the functional differences in the brain-relevant HDL activities evaluated in this study. 
a

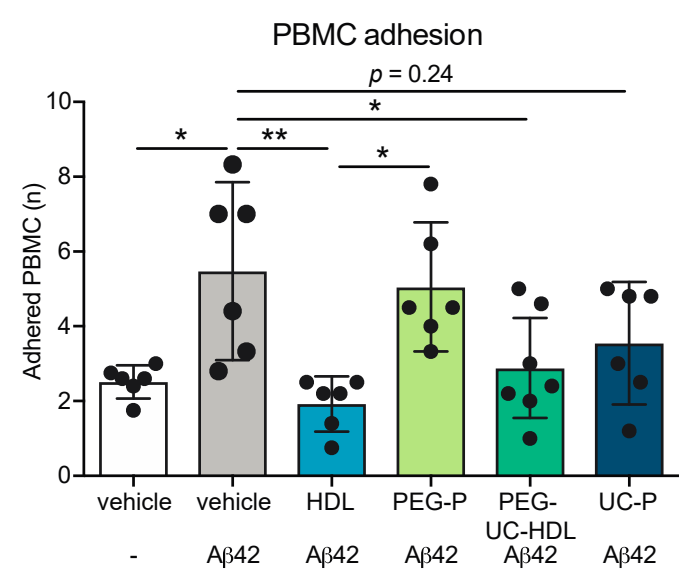

b

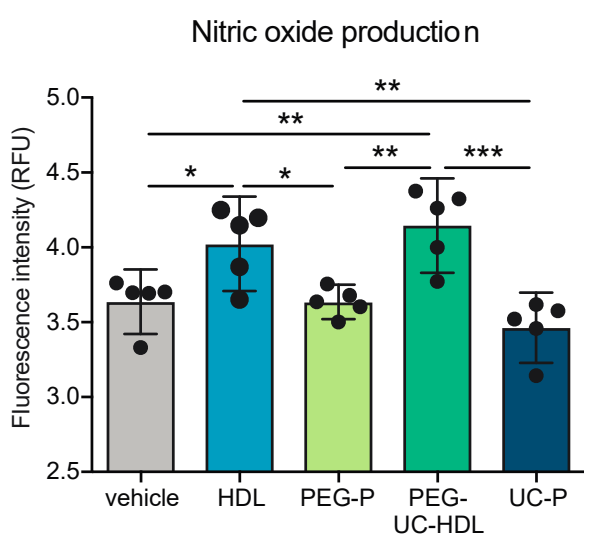

Figure 5. Non-HDL plasma components interfere with some anti-inflammatory activities of HDL is apoB-depleted plasma. (a) Adhesion of fluorescently PBMC to hCMEC/d3 was measured using fluorescent microscopy on cells that were pretreated with HDL $(100 \mu \mathrm{g} / \mathrm{mL}$ protein, $5 \mathrm{mg} / \mathrm{dL}$ HDL-C) or apoB-depleted plasma ( $5 \mathrm{mg} / \mathrm{dL}$ HDL-C) fractions for $2 \mathrm{~h}$ then stimulated with A $\beta 42(0.1 \mu \mathrm{mol} / \mathrm{L})$ for $3 \mathrm{~h}$; (b) nitric oxide production in unstimulated hCMEC/d3 was measured using the DAF-2 DA and HDL or apoB-depleted plasma fractions treatment for $4 \mathrm{~h}$. Scatter plots represent independent experiments with mean $\pm 95 \%$ confidence interval. ${ }^{*} p<0.05,{ }^{* *} p<0.01,{ }^{* *} p<0.001$, or exact $p$-value by one-way ANOVA with Tukey's multiple comparisons test. RFU: relative fluorescence units; HDL: high-density lipoproteins isolated by sequential density gradient ultracentrifugation; PEG-P: apoB-depleted plasma by polyethylene glycol precipitation; PEG-UC: combination method of PEG precipitation and ultracentrifugation HDL; UC-P: 1 step ultracentrifugation HDL; PBMC: peripheral blood mononuclear cell; A $\beta 42$ : amyloid beta 42; DAF-2 DA: 4,5-diaminofluorescein diacetate.

\section{Discussion}

It is well-known that HDL composition varies according to the method by which this heterogeneous lipoprotein subclass is isolated [25-27]. It is also increasingly clear that HDL function may be more relevant than HDL-C levels when predicting coronary artery disease risk [17-19]. Although Mendelian randomization studies showed that high HDL-C levels do not causally contribute to reduced coronary artery disease risk [28], HDL may still have cerebrovascular functions that lower AD risk. Aging, T2DM, hypertension, and hypercholesterolemia are established AD risk factors and all can be associated with reduced HDL function [29]. Changes in HDL functions such as attenuation of inflammation in vascular diseases may partly account for the association between these diseases and AD risk. Supporting this concept is the observation that HDL isolated from AD patients displays reduced cholesterol efflux capacity and a reduced ability to suppress TNF $\alpha$-induced intercellular adhesion molecule 1 (ICAM-1) expression in EC compared to HDL isolated from cognitively healthy individuals [30]. Whether HDL has additional functions that are more specific to cerebrovascular health in $\mathrm{AD}$ is now an important question.

The cerebrovascular system has an important role in clearing $A \beta$ from the brain across the BBB or through perivascular clearance along the artery wall [31]. Dysfunction in the vascular clearance of $A \beta$ is common in $A D$ and is thought to contribute to the deposition of $A \beta$ within cerebral arteries known as CAA [32]. Modeling A $\beta$ clearance and CAA formation with $3 \mathrm{D}$ bioengineered arteries allowed us to discover that HDL circulated through the vessel lumen suppresses $A \beta$ accumulation in the vessel wall and tends to increase $A \beta$ recovery in the luminal circulating media [15]. Furthermore, it has been suggested that the exposure of vascular cells to $A \beta$ during clearance from the brain may result in vascular inflammation [21-23]. We confirmed that $A \beta 42$ has inflammatory effects in 3D bioengineered arteries and 2D EC cultures and further found that HDL suppressed this A $\beta$-induced EC activation [16]. Importantly, it is increasingly recognized that in vitro experimental data obtained 
using traditional 2D cell culture methods may be of limited translational relevance [33]. As our 3D bioengineered vascular model maintains the anatomical and physiological complexity of a native vessel, it may be a better model to investigate HDL function under more physiological conditions. However, as our 3D models requires substantial sample volume, this study was designed to optimize preparation method to prepare for studies using clinical biospecimens from patients with known AD risk factors such as age and cardiometabolic diseases.

Density gradient ultracentrifugation is the method by which lipoprotein subclasses were first discovered in human plasma, and remains a key method in their isolation today including use in several clinical studies [24,34]. However, this method has several established drawbacks including loss of some HDL proteins, such as apoE and apoA-I, due to high shear forces [26] and the potential redistribution of apolipoproteins due to high ionic strength during isolation and the subsequent desalting process [35]. Indeed, we observed that apoE levels were significantly lower in HDL isolated by ultracentrifugation compared to plasma and apoB-depleted plasma when normalized to HDL-C content (Figure S3c). Ultracentrifugation also requires a substantial volume of starting material, is relatively costly and is low throughput. A higher-throughput method of HDL isolation uses the neutral polymer PEG to precipitate apoB-containing, non-HDL lipoproteins from plasma. ApoB-depletion using PEG is attractive for clinical studies as it is cost effective, high throughput and requires minimal specimen volume. ApoB-depleted plasma has been used previously in clinical studies to evaluate cholesterol efflux capacity and demonstrates a similar maximal efflux capacity compared to HDL isolated by ultracentrifugation [36], which we confirmed here (Figure S3f). Nevertheless, it is important to note that apoB-depleted plasma is a heterogeneous preparation that retains proteins such as albumin and globulins as well as other factors that may affect certain functional assays. Furthermore, Davidson et. al. recently evaluated the use of PEG-precipitation in comparison to other precipitation reagents and found that while PEG-precipitation does not drastically alter the phospholipid or cholesterol content of particles isolated in the HDL size range compared to its profile in native serum, a finding that we also confirmed (Figure S3a,d), the distribution of apoA-I and apoE and the cholesterol efflux capacity of specific HDL fractions was altered. Therefore, caution was suggested in using PEG-precipitation in HDL functional assays [25].

Here we show that sequential ultracentrifugation is superior to PEG-precipitation as a method to isolate HDL particles for assays of cerebrovascular functions relevant to AD. Where sample volume may be too small for ultracentrifugation, apoB-depleted plasma functioned equally as well as ultracentrifuge-isolated HDL with respect to CAA. As CAA cannot yet be evaluated in live humans, assaying HDL in 3D bioengineered arteries may provide an unprecedented proxy of human CAA risk in vivo. The ability to use apoB-depleted plasma instead of HDL isolated by ultracentrifugation may streamline efforts to evaluate the anti-CAA effects of HDL in clinical specimens. However, apoB-depleted plasma is not suitable for other AD-relevant assays, including promoting A $\beta$ transport through the wall of 3D bioengineered arteries or NO production. Several potential mechanisms may explain the discrepancy in the effects against accumulation in the vessel wall compared to clearance into the lumen. For example, apoB-depleted plasma may prevent the entrance of abluminal A $\beta 42$ into the vessel wall while HDL promotes the removal of $A \beta 42$ from the vessel wall and clearance into the lumen. It is also possible that the presence of extensive plasma proteins in the apoB-depleted plasma circulating in the luminal media may mask the $A \beta 42$ epitope and reduce its detection in luminal media by ELISA. Finally, we found that apoB-depleted plasma did not recapitulate the ability of HDL isolated by ultracentrifugation to suppress $\mathrm{A} \beta$-induced vascular inflammation in $2 \mathrm{D}$ and $3 \mathrm{D}$ models or to induce NO production in brain-derived EC. Nevertheless, we demonstrated here that TNF $\alpha$-induced EC inflammation is reduced by apoB-depleted plasma similarly to HDL isolated by ultracentrifugation, which is of interest for investigators studying cardiovascular disease.

The discrepancy between ultracentrifuge-isolated HDL and apoB-depleted plasma in these specific functions cannot be attributed to residual PEG in the latter preparation. This is because combining PEG precipitation with a single ultracentrifugation step produces a preparation with similar functions 
to the traditional ultracentrifugation method. Instead, the functional differences in PEG-precipitated plasma may result from remaining non-HDL plasma proteins that potentially interfere with certain HDL functions. That PEG-precipitated plasma and plasma subjected to a single ultracentrifugation to remove apoB-containing lipoproteins perform similarly in the assays tested here supports the interference hypothesis. It is also possible that the functional differences observed between the two plasma preparations could be explained by a loss or redistribution of apolipoproteins resulting from the high shear and ionic forces used during $\mathrm{KBr}$ density gradient ultracentrifugation [35,37]. In future, the use of $\mathrm{D}_{2} \mathrm{O}$ /sucrose as a density agent instead of $\mathrm{KBr}$, as investigated by Ståhlman et al. [35], could help to investigate the effect of ionic strength on the functions of isolated HDL.

There are several limitations to this study. First, the donors who participated in this study were young and healthy, and we anticipate that at least some of the AD-relevant functions assessed here may be altered by aging or comorbid conditions associated with increased AD risk, which are associated with increased blood inflammatory markers [38]; second, a relatively small number of functional tests assayed in this study were selected for their potential relevance to AD or cerebrovascular health. Furthermore, HDL was only assessed at a single concentration, which corresponds to the most frequent concentration used in studies evaluating HDL functions in vitro [24,30,34,39-41], therefore presenting the possibility of effect saturations that may mask subtle functional differences by isolation method. Similarly, we evaluated only two common HDL isolation methods. Other HDL isolation methods such as immunoaffinity chromatography or fast protein liquid chromatography were not pursued here as these methods do not provide sufficient HDL for the cell-based functional assays in this study. Another limitation is that we chose to normalize preparations to HDL-C concentrations. HDL and apoB-depleted plasma preparations could alternatively have been normalized to apoA-I concentrations, however, this was avoided due to potential loss of apoA-I from HDL particles during ultracentrifugation [26]. HDL particle number is another method of normalization, which could be used in the future. Although normalization to the initial plasma volume could have been used, potential variances in product loss between isolation methods was a concern. Finally, future mass-spectrometry studies will be needed to define the compositional differences between HDL preparations and understand their association to each of the AD-relevant HDL functions tested here.

In summary, we report here that apoB-depleted plasma can be used to evaluate select HDL functions relevant to cerebrovascular health, but that ultracentrifugation is the preferred method to isolate HDL that exhibits at least 4 distinct AD-relevant vasoprotective functions. First, HDL delays $A \beta$ fibrillization in a cell-free assay; second, HDL induces NO production in a process that is inhibited by co-treatment with large amounts of non-HDL plasma components; third, HDL inhibits A $\beta$-induced endothelial activation in brain-derived EC; fourth, HDL may protect against AD by preventing A $\beta$ accumulation within cerebral vessels.

\section{Materials and Methods}

\subsection{Blood Collection}

All experiments were conducted under an approved protocol (UBC Clinical Ethics Research Board H14-03357, 10 Novermber 2015). Non-fasting blood was obtained from healthy, normolipidemic male or female volunteers aged 20 to 35 years in dipotassium ethylenediaminetetraacetic acid $\left(\mathrm{K}_{2}\right.$-EDTA) coated plasma vacutainer tubes (BD Biosciences, San Jose, CA, USA) by venipuncture after receipt of informed consent. Plasma was isolated from whole blood by centrifugation at $1500 \times g$ for $10 \mathrm{~min}$. Additional plasma was obtained from the Network Centre for Applied Development (netCAD) through the Canadian Blood Services under an approved protocol (Canadian Blood Services REB 2016.015, nc0027, 25 October 2017). Plasma was either immediately processed to isolate lipoproteins or aliquoted and stored at $-20^{\circ} \mathrm{C}$ until use. Whole blood for PBMC isolation was collected from healthy volunteers as described above and PBMC were by isolated by centrifugation on a continuous density gradient (Lymphoprep, StemCell, Vancouver, BC, Canada) following the manufacturer's directions. 


\subsection{HDL Isolation}

Except where indicated otherwise, HDL was isolated from plasma by 2 step sequential potassium bromide (KBr) gradient density ultracentrifugation [16] (Figure S2a) and apoB-depleted plasma was produced by polyethylene glycol (PEG) precipitation [25] (Figure S2b). HDL and apoB-depleted plasma were dialyzed in dialysis buffer $(150 \mathrm{mmol} / \mathrm{L} \mathrm{NaCl}, 0.3 \mathrm{mmol} / \mathrm{L} \mathrm{EDTA}, \mathrm{pH} 7.4)$ for 2 days at $4{ }^{\circ} \mathrm{C}$, with the buffer refreshed 4 times.

For selected experiments, two additional isolation methods were used. First, PEG-precipitation was used to remove apoB-containing lipoproteins after which the density of the supernatant was adjusted to $1.21 \mathrm{~g} / \mathrm{mL}$ and subjected to a single ultracentrifugation step to remove other plasma proteins from the HDL fraction. The upper fraction containing HDL was collected and dialyzed as above (Figure S2c); second, a single step ultracentrifugation was performed by adjusting plasma density to $1.063 \mathrm{~g} / \mathrm{mL}$ with $\mathrm{KBr}$ and centrifuging at $160,000 \times \mathrm{g}$ for $16 \mathrm{~h}$ at $14{ }^{\circ} \mathrm{C}$. The upper fraction containing non-HDL lipoproteins was removed and the bottom fraction containing HDL and other plasma proteins was collected and dialyzed as above (Figure S2d).

\subsection{Enzyme-Linked Immunosorbent Assay (ELISA)}

ApoA-I levels were quantified by ELISA (Mabtech, Cincinnati, OH, USA, 3710-1HP-2) following the manufacturer's directions with a 50,000-fold dilution in the supplied dilution buffer. ApoE levels were quantified using an in-house sandwich ELISA [42]. ELISA data for apoA-I and apoE were normalized to HDL-C levels for both HDL or apoB-depleted plasma preparations.

\subsection{Cell Culture}

Human cerebral microvascular endothelial cells (hCMEC/d3) (Fisher, Hampton, NH, USA) passage 30-40 were maintained in complete endothelium growth medium 2 (EGM2) (Lonza, Visp Switzerland) with $2 \%$ foetal bovine serum (FBS) (Invitrogen, Carlsbad, CA, USA) in a humidified incubator at $37^{\circ} \mathrm{C}$ at $5 \%$ carbon dioxide $\left(\mathrm{CO}_{2}\right)$. Murine RAW 264.7 macrophages (ATCC, Manassas, VA, USA) were maintained in Dulbecco's modified eagle medium (DMEM) (Invitrogen) containing $10 \%$ heat-inactivated FBS (Invitrogen) in a humidified incubator at $37^{\circ} \mathrm{C}$ at $5 \% \mathrm{CO}_{2}$.

\subsection{Fabrication of 3D Bioengineered Vessels}

3D bioengineered vessels composed of human cells were grown on a scaffold and maintained in culture under dynamic pulsatile flow as described [15]. Briefly, $10 \times 2 \mathrm{~mm}$ tubular scaffolds were formed from non-woven polyglycolic-acid meshes coated with $1.75 \%$ polycaprolactone $(80 \mathrm{kDa}$, Millipore Sigma, Burlington, MA, USA) in tetrahydrofuran (Millipore Sigma). Human umbilical smooth muscle cells (SMC) and endothelial cells (HUVEC) were isolated as described [43] under UBC ethics protocol H14-03372; $2 \times 10^{6} \mathrm{SMC} / \mathrm{cm}^{2}$ were seeded within sterilized and equilibrated scaffolds followed by 3 days in static culture and 7 days of culture under dynamic pulsatile flow in the bioreactor. $1 \times 10^{6} \mathrm{HUVEC} / \mathrm{cm}^{2}$ were then seeded within the scaffolds followed by culture in static conditions for 5 days then 10 days under dynamic pulsatile flow in the bioreactor. Vessels were characterized histologically as described [15]. Briefly, O.C.T. (ThermoFisher, Waltham, MA, USA) embedded vessels were sectioned at $20 \mu \mathrm{m}$ on a cryostat (Leica, Germany) then rehydrated with phosphate buffered saline (PBS), fixed for $20 \mathrm{~min}$ in 4\% paraformaldehyde (PFA), washed in Tris- $\mathrm{HCl}(0.5 \mathrm{~mol} / \mathrm{L}, \mathrm{pH} 7.6)$, and blocked in 5\% goat serum, 1\% BSA in PBS. Immunofluorescence was performed to visualize HUVEC and SMC by incubating in primary antibodies for CD31 (Biolegend, San Diego, CA, USA, WM59, 1:50) or smooth muscle actin (Millipore Sigma, 1A4, 1:200) overnight followed by washing and incubation in secondary anti-rabbit or anti-mouse Alexa-488 or Alexa-594 antibodies (Invitrogen) for $45 \mathrm{~min}$ at room temperature. Sections were washed with PBS then mounted in Prolong antifade containing DAPI (ThermoFisher) and imaged on an inverted fluorescent microscope (Zeiss, Oberkochen, Germany). 


\subsection{Nitric Oxide Production}

hCMEC/d3 were seeded at $5 \times 10^{5}$ cells/well in 6-well plates (Fisher, Hampton, NH, USA) and cultured in complete EGM2 for 3 days before serum starvation $(0.2 \%$ FBS $)$ for $16 \mathrm{~h}$. Cells were treated with $50 \mu \mathrm{g} / \mathrm{mL}$ HDL isolated by ultracentrifugation or an equivalent amount of apoB-depleted plasma based on HDL-C concentration, both from the same plasma donor, along with $1 \mu \mathrm{mol} / \mathrm{L}$ of 4,5-diaminofluorescein diacetate (DAF2-DA, Cayman Chemical, Ann Arbor, MI, USA) in EGM2 lacking vascular endothelial growth factor (VEGF) and $0.2 \%$ FBS. After $4 \mathrm{~h}$, cells were washed with PBS and trypsinized for $5 \mathrm{~min}$. Triazolofluorescein fluorescence was measured (excitation $485 \mathrm{~nm}$, emission $538 \mathrm{~nm}$ ) using an Infinite M200Pro plate reader (Tecan, Männedorf, Switzerland).

\subsection{Preparation of $A \beta$ Monomers}

Recombinant A $\beta 42$ peptides (California Peptide, Napa, CA, USA) were dissolved in ice-cold hexafluoroisopropanol (HFIP, Millipore Sigma) and aliquoted. HFIP was removed by evaporation overnight and the $A \beta$ film was stored at $-20^{\circ} \mathrm{C}$ until use. The film was reconstituted in DMSO to $5 \mathrm{mmol} / \mathrm{L}$ followed by a further dilution to $100 \mu \mathrm{mol} / \mathrm{L}$ in FBS free EBM2 containing $0.2 \%$ BSA immediately before use.

\subsection{Anti-Inflammatory Function}

hCMEC/d3 were seeded at $1 \times 10^{5}$ cells/well in 24-well plates (Fisher, Hampton, NH, USA) for 3 days until confluent. Cells were primed for $2 \mathrm{~h}$ with $50 \mu \mathrm{g} / \mathrm{mL}$ HDL isolated by ultracentrifugation or an equivalent amount of apoB-depleted plasma based on cholesterol concentration, both from the same plasma donor, in FBS-free EGM2 containing $0.2 \%$ BSA then treated with $1 \mathrm{ng} / \mathrm{mL}$ TNF $\alpha$ (Preprotech, Rocky Hill, NJ, USA) or $0.1 \mu \mathrm{mol} / \mathrm{L} A \beta$ monomers for $3 \mathrm{~h}$. The dose of A $\beta$ used here was selected based on our previous work investigating the response of PBMC adhesion to increasing doses of $A \beta$ [16]. PBMC previously labeled for $30 \mathrm{~min}$ with Cell-Tracker Red (Invitrogen) following the manufacturer's instructions, were added at a density of $5 \times 10^{5}$ cells/well and allowed to adhere for $3 \mathrm{~h}$. Notably, as the media containing the treatments was not removed between steps of this assay, the final conditioned media contained HDL or apoB-depleted plasma in combination with A $\beta 42$ or TNF $\alpha$ and labelled PBMC. Cells were extensively washed with PBS, fixed with $4 \%$ PFA for $15 \mathrm{~min}$, washed with PBS and counterstained with 4,6-diamidino-2-phenylindole (DAPI, Sigma Millipore) (100 ng/mL). Adhered PBMC were imaged using an inverted fluorescent microscope (Zeiss) and counted in 5 random squares of $7.84 \mathrm{~mm}^{2}$ using ImageJ (National Institutes of Health, Bethesda, MD, USA).

\subsection{Cell-Free Assay of A $\beta$ Fibrillization}

The formation of $A \beta$ fibrils was measured using Thioflavin $T$, an indicator of $\beta$-sheet formation, in a cell-free assay. Infinite M2000 Pro plate reader (Tecan). $10 \mu \mathrm{mol} / \mathrm{L}$ monomeric A $\beta 42$ were incubated in a buffer consisting of Thioflavin- $\mathrm{T}(20 \mathrm{mmol} / \mathrm{L}$ in $150 \mathrm{mmol} / \mathrm{L} \mathrm{NaCl}$ and $5 \mu \mathrm{mol} / \mathrm{L}$ of HEPES at $\mathrm{pH}$ 7.4), with and without $0.1 \mathrm{mg} / \mathrm{mL}$ HDL isolated by ultracentrifugation or an equivalent amount of apoB-depleted plasma based on HDL-C concentration, both from the same donor in a 96-well, black plate. The plate was kept at $37^{\circ} \mathrm{C}$ and subjected to orbital shaking for $20 \mathrm{~s}$ every $5 \mathrm{~min}$ using an Infinite M2000 Pro plate reader. Fluorescence intensity was measured every $5 \mathrm{~min}$ for $12 \mathrm{~h}$ in total (440/490 $\mathrm{nm}$ excitation/emission).

\subsection{Amyloid Beta Clearance and Accumulation Assays Using Engineered Vessels}

A $\beta 42$ monomers were injected within the tissue chamber (the "brain side") to a final concentration of $1 \mu \mathrm{mol} / \mathrm{L}$, a dose chosen based on a previous dose-response experiment [15]. Immediately afterward, the bioreactor media was substituted with complete EGM2 media ( $2 \%$ FBS) containing $200 \mu \mathrm{g} / \mathrm{mL}$ HDL isolated by ultracentrifugation or an equivalent amount of apoB-depleted plasma based on cholesterol concentration, both from the same plasma donor. Luminal medium was collected from 
the circulation chamber (the "blood side") from $5 \mathrm{~min}$ to $4 \mathrm{~h}$ and $24 \mathrm{~h}$ after treatment. At $24 \mathrm{~h}$ bioengineered tissues were collected and lysed in RIPA buffer $(10 \mathrm{mM}$ Tris pH 7.4, $150 \mathrm{mM} \mathrm{NaCl}, 1.0 \%$ NP-40, 1.0\% sodium deoxycholate, $0.1 \%$ SDS and cOmplete protease inhibitor with EDTA (Roche, Basel, Switzerland)). A $\beta 42$ (KHB3482, ThermoFisher) was quantified in circulating media and tissue lysates using commercial ELISA and tissue concentrations were normalized to total protein content measured by BCA protein assay kit (ThermoFisher).

\subsection{Amyloid Beta Induced Monocyte Adhesion Using Engineered Vessels}

As above, A $\beta 42$ monomers were injected within the tissue chamber (the "brain side") to a final concentration of $1 \mu \mathrm{mol} / \mathrm{L}$, a dose selected from a previous dose-response experiment [16]. Immediately afterward, the bioreactor media was substituted with complete EGM2 media (2\% FBS) containing $200 \mu \mathrm{g} / \mathrm{mL}$ HDL isolated by ultracentrifugation or an equivalent amount of apoB-depleted plasma based on cholesterol concentration, both from the same plasma donor. After $21 \mathrm{~h}$, Cell-Tracker Red labelled THP-1 monocytes were added to the circulating media ( 1 million cells $/ \mathrm{mL}$ ) and allowed to adhere for $3 \mathrm{~h}$. Bioengineered tissues were then collected, fixed in $4 \%$ PFA in PBS, rinsed in PBS, and the entire tissue thickness was imaged using a SP8 confocal microscope (Leica). Adhered monocytes were counted in 3 random squares of $1.23 \mathrm{~mm}^{2}$.

\subsection{Characterization of HDL Preparations}

The protein concentration of HDL was quantified using the Pierce BCA Protein Assay Kit (ThermoFisher, Waltham, MA, USA) following the manufacturer's directions. HDL cholesterol (HDL-C), phospholipid, free and esterified cholesterol, and triglycerides were quantified using commercially available kits (Wako Diagnostics, Mountain View, CA, USA) following the manufacturer's directions.

\subsection{Gel Electrophoresis}

HDL, apoB-depleted plasma, and total plasma were analysed using native and sodium dodecyl sulphate (SDS) polyacrylamide gel electrophoresis. HDL (10 $\mu \mathrm{g}$ of protein) and an equivalent amount of apoB-depleted plasma based on HDL-C concentration, both from the same plasma donor, were separated by electrophoresis, using $6 \%$ acrylamide for native and $10 \%$ for denaturing, respectively. To visualize all proteins, gels were washed in water, stained with Coomassie R-250 ( $0.1 \%$ Coomassie R-250, 40\% methanol, $10 \%$ acetic acid) overnight, destained in a solution of $10 \%$ methanol and 7.5\% acetic acid in water for at least $2 \mathrm{~h}$, and imaged using a ChemiDoc MP imager (Bio-Rad, Hercules, CA, USA).

\subsection{Statistical Analysis}

Statistical analyses were performed with GraphPad Prism 6.05 software (GraphPad, San Diego, CA, USA) and $p<0.05$ was considered significant. Data were obtained from at least three independent experiments with 3 independent donors. Raw data, or log-transformed data in the case of the NO production assay due to non-normality, were analyzed by one-way analysis of variance (ANOVA) with Tukey's multiple comparison test or two-way ANOVA in the case of A $\beta 42$ recovery into the circulation of engineered vessels. Data are represented as scatter plots overlaid with calculated means $\pm 95 \%$ confidence interval using error bars. Analysis of $A \beta$ fibrillization in the cell-free thioflavin $\mathrm{T}$ assay was performed after fitting to a Boltzman sigmoidal curve using GraphPad Prism 6.05 software then extracting $\mathrm{t}_{50}$ and curve maximum from the model.

\section{Conclusions}

A relationship between HDL and brain health has emerged with the discovery of several novel, brain-relevant vasoprotective functions of HDL coupled with epidemiological and animal model data 
supporting a protective role for HDL particularly with respect to CAA. Evaluation of AD-relevant HDL functions in clinical populations now becomes an important question. To prepare for studies using clinical specimens, many of which are banked and may be of limited volume, it is important to optimize the method by which HDL is prepared, which will determine cost, volume of plasma required, throughput, and the repertoire of the functions that can be studied in vitro. We compared HDL isolated by density gradient ultracentrifugation to apoB-depleted plasma produced by PEG-precipitation in several novel assays of HDL function and found that apoB-depleted plasma is suitable to evaluate some, but not all, of the beneficial functions of HDL that are relevant to AD. Specifically, apoB-depleted plasma can be used in assays of suppressing $A \beta$ accumulation in bioengineered vessels, preventing $\mathrm{A} \beta$ fibrillization, and suppressing TNF $\alpha$-induced vascular inflammation. However, apoB-depleted plasma does not replicate the ability of HDL isolated by ultracentrifugation to suppress $A \beta$-induced vascular inflammation, improve $A \beta$ vascular clearance, or induce endothelial NO production.

Supplementary Materials: Supplementary materials can be found at http:/ /www.mdpi.com/1422-0067/20/3/ $462 /$ s1.

Author Contributions: Conceptualization: E.B.B., J.R. and C.L.W.; Formal Analysis: E.B.B.; Funding acquisition: J.R. and C.L.W.; Investigation: E.B.B., M.G., H.K.C., E.M.M., A.A. and J.R.; Methodology: E.B.B., J.R. and C.L.W.; Supervision: J.R. and C.L.W.; Visualization: E.B.B. and J.R.; Writing-original draft: E.B.B., J.R. and C.L.W; Writing-review and editing: E.B.B., M.G., H.K.C., E.M.M., J.R. and C.L.W.

Acknowledgments: This study was supported by a Canadian Institutes of Health Research (CIHR) Doctoral Award and a UBC Four Year Graduate Fellowship to EBB. Fellowship and operating funding to JR from the BrightFocus Foundation, the University of British Columbia (Bluma Tischler), the Michael Smith Foundation for Health Research, and Weston Brain Institute, and operating funding to CLW through the CIHR-supported Canadian Consortium for Neurodegeneration in Aging (CCNA) and a donation from the Jack Brown and Family Foundation.

Conflicts of Interest: The authors declare no conflict of interest.

\section{References}

1. Patterson, C. World Alzheimer Report 2018. The State of the Art of Dementia Research: New Frontiers; Alzheimer's Disease International: London, UK, 2018.

2. Attems, J.; Jellinger, K.A. The overlap between vascular disease and Alzheimer's disease-Lessons from pathology. BMC Med. 2014, 12, 206. [CrossRef] [PubMed]

3. Cupino, T.L.; Zabel, M.K. Alzheimer's Silent Partner: Cerebral Amyloid Angiopathy. Transl. Stoke Res. 2014, 5, 330-337. [CrossRef]

4. Corriveau, R.A.; Gladman, J.T.; Jeon, S.; Babcock, D.; Bennett, D.A.; Carmichael, S.T.; Dickinson, S.L.; Emr, M.; Fillit, H.; Greenberg, S.M.; et al. Alzheimer's Disease-Related Dementias Summit 2016: National research priorities. Am. Acad. Neurol. 2017, 89, 2381-2391. [CrossRef]

5. Crichton, G.E.; Elias, M.F.; Davey, A.; Sullivan, K.J.; Robbins, M.A. Higher HDL cholesterol is associated with better cognitive function: The Maine-Syracuse study. J. Int. Neuropsychol. Soc. 2014, 20, 961-970. [CrossRef] [PubMed]

6. Bates, K.A.; Sohrabi, H.R.; Rainey-Smith, S.R.; Weinborn, M.; Bucks, R.S.; Rodrigues, M.; Beilby, J.; Howard, M.; Taddei, K.; Martins, G.; et al. Serum high-density lipoprotein is associated with better cognitive function in a cross-sectional study of aging women. Int. J. Neurosci. 2017, 127, 243-252. [CrossRef] [PubMed]

7. Tortelli, R.; Lozupone, M.; Guerra, V.; Rosaria, M.; Imbimbo, B.P.; Capozzo, R.; Grasso, A.; Tursi, M.; di Dio, C.; Sardone, R.; et al. Midlife Metabolic Profile and the Risk of Late-Life Cognitive Decline. J. Alzheimer's Dis. 2017, 59, 121-130. [CrossRef]

8. Ma, C.; Yin, Z.; Zhu, P.; Luo, J.; Shi, X.; Gao, X. Blood cholesterol in late-life and cognitive decline: A longitudinal study of the Chinese elderly. Mol. Neurobiol. Mol. Neurodegener. 2017, 12, 24. [CrossRef]

9. Kim, W.; He, Y.; Phan, K.; Ahmed, R.; Rye, K.; Piguet, O.; Hodges, J.R.; Halliday, G.M. Altered High Density Lipoprotein Composition in Behavioral Variant Frontotemporal Dementia. Front. Neurosci. 2018, $12,847$. [CrossRef] 
10. Lefterov, I.; Fitz, N.F.; Cronican, A.A.; Fogg, A.; Lefterov, P.; Kodali, R.; Wetzel, R.; Koldamova, R. Apolipoprotein A-I Deficiency Increases Cerebral Amyloid Angiopathy and Cognitive Deficits in APP/PS1DeltaE9 Mice. J. Biol. Chem. 2010, 285, 36945-36957. [CrossRef]

11. Lewis, T.L.; Cao, D.; Lu, H.; Mans, R.A.; Su, Y.R.; Jungbauer, L.; Linton, M.F.; Fazio, S.; LaDu, M.J.; Li, L. Overexpression of Human Apolipoprotein A-I Preserves Cognitive Function and Attenuates Neuroinflammation and Cerebral Amyloid Angiopathy in a Mouse Model of Alzheimer. J. Biol. Chem. 2010, 285, 36958-36968. [CrossRef]

12. Handattu, S.P.; Garber, D.W.; Monroe, C.E.; van Groen, T.; Kadish, I.; Nayyar, G.; Cao, D. Oral apolipoprotein A-I mimetic peptide improves cognitive function and reduces amyloid burden in a mouse model of Alzheimer's disease. Neurobiol. Dis. 2009, 34, 525-534. [CrossRef] [PubMed]

13. Robert, J.; Stukas, S.; Button, E.; Hang, W.; Lee, M.; Fan, J.; Wilkinson, A.; Kulic, I.; Wright, S.D. Wellington CL. Reconstituted high-density lipoproteins acutely reduce soluble brain A $\beta$ levels in symptomatic APP/PS1 mice. Biochim. Biophys. Acta 2015, 1862, 1027-1036. [CrossRef] [PubMed]

14. Fernández-de-Retana, S.; Cano-Sarabia, M.; Marazuela, P.; Sánchez-Quesada, J.L.; Garcia-Leon, A.; Montañola, A.; Montaner, J.; Maspoch, D.; Hernández-Guillamon, M. Characterization of ApoJ-reconstituted high-density lipoprotein (rHDL) nanodisc for the potential treatment of cerebral $\beta$-amyloidosis. Sci. Rep. 2017, 7, 14637. [CrossRef] [PubMed]

15. Robert, J.; Button, E.B.; Yuen, B.; Gilmour, M.; Kang, K.; Bahrabadi, A.; Stukas, S.; Zhao, W.; Kulic, I.; Wellington, C.L. Clearance of beta-amyloid is facilitated by apolipoprotein E and circulating high- density lipoproteins in bioengineered human vessels. eLife 2017, 6, e29595. [CrossRef]

16. Robert, J.; Button, E.B.; Stukas, S.; Boyce, G.K.; Gibbs, E.; Cowan, C.M.; Gilmour, M.; Cheng, W.H.; Soo, S.K.; Yuen, B.; et al. High-density lipoproteins suppress A $\beta$-induced PBMC adhesion to human endothelial cells in bioengineered vessels and in monoculture. Mol. Neurodegener. 2017, 12, 60. [CrossRef] [PubMed]

17. Rohatgi, A.; Khera, A.V.; Berry, J.D.; Givens, E.G.; Ayers, C.R.; Wedin, K.E.; Neeland, I.J.; Yuganna, I.S.; Rader, D.R.; de Lemos, J.A.; et al. HDL Cholesterol Efflux Capacity and Incident Cardiovascular Events. N. Engl. J. Med. 2014, 371, 2383-2393. [CrossRef] [PubMed]

18. Saleheen, D.; Scott, R.; Javad, S.; Zhao, W.; Rodrigues, A.; Picataggi, A.; Lukmanova, D.; Muchsavage, M.L.; Luben, R.; Billheimer, J.; et al. Association of HDL cholesterol efflux capacity with incident coronary heart disease events: A prospective case-control study. Lancet Diabetes Endocrinol. 2015, 3, 507-513. [CrossRef]

19. Khera, A.V.; Cuchel, M.; de la Llera Moya, M.; Rodrigues, A.; Burke, M.; Jafri, K.; French, B.C.; Phillips, J.A.; Mucksavage, M.L.; Wilensky, R.L.; et al. Cholesterol Efflux Capacity, High-Density Lipoprotein Function, and Atherosclerosis. N. Engl. J. Med. 2011, 364, 127-135. [CrossRef]

20. Genest, J.; McPherson, R.; Frohlich, J.; Anderson, T.; Campbell, N.; Carpentier, A.; Couture, P.; Dufour, R.; Fodor, G.; Francis, G.A.; et al. 2009 Canadian Cardiovascular Society/Canadian guidelines for the diagnosis and treatment of dyslipidemia and prevention of cardiovascular disease in the adult-2009 recommendations. Can. J. Cardiol. 2009, 25, 567-579. [CrossRef]

21. Giri, R.; Selvaraj, S.; Miller, C.A.; Hofman, F.; Yan, S.D.; Stern, D.; Zlokovic, B.V.; Kalra, V.K. Effect of endothelial cell polarity on $\beta$-amyloid-induced migration of monocytes across normal and AD endothelium. Am. J. Physiol. Physiol. 2002, 283, C895-904. [CrossRef]

22. Truran, S.; Weissig, V.; Madine, J.; Davies, H.A.; Guzman-Villanueva, D.; Franco, D.A.; Karamanova, N.; Burciu, C.; Serrano, G.; Beach, T.G.; et al. Nanoliposomes protect against human arteriole endothelial dysfunction induced by $\beta$-amyloid peptide. J. Cereb. Blood Flow Metab. 2016, 36, 405-412. [CrossRef] [PubMed]

23. Lamoke, F.; Mazzone, V.; Persichini, T.; Maraschi, A.; Harris, M.B.; Venema, R.C.; Colasanti, M.; Gliozzi, M.; Muscoli, C.; Bartoli, M.; et al. Amyloid $\beta$ peptide-induced inhibition of endothelial nitric oxide production involves oxidative stress-mediated constitutive eNOS/HSP90 interaction and disruption of agonist-mediated Akt activation. J. Neuroinflamm. 2015, 12, 84. [CrossRef] [PubMed]

24. Besler, C.; Heinrich, K.; Rohrer, L.; Doerries, C.; Riwanto, M.; Shih, D.M.; Chroni, A.; Yonekawa, K.; Stein, S.; Schaefer, N.; et al. Mechanisms underlying adverse effects of HDL on eNOS-activating pathways in patients with coronary artery disease. J. Clin. Investig. 2011, 121, 2693-2708. [CrossRef] [PubMed]

25. Davidson, W.S.; Heink, A.; Sexmith, H.; Melchior, J.T.; Gordon, S.M.; Kuklenyik, Z.; Woollett, L.; Barr, J.R.; Jones, J.I.; Toth, C.A.; et al. The effects of apolipoprotein B depletion on HDL subspecies composition and function. J. Lipid Res. 2016, 57, 674-686. [CrossRef] [PubMed] 
26. Kunitake, S.T.; Kane, J.P. Factors affecting the integrity of high density lipoproteins in the ultracentrifuge. J. Lipid Res. 1982, 23, 936-940. [PubMed]

27. Van't Hooft, F.; Havel, R. Metabolism of Apolipoprotein E in Plasma High Density Lipoproteins from Normal and Cholesterol-fed Rats. J. Biol. Chem. 1982, 257, 10996-11001. [PubMed]

28. Holmes, M.V.; Asselbergs, F.W.; Palmer, T.M.; Drenos, F.; Lanktree, M.B.; Nelson, C.P.; Dale, C.E.; Padmanabhan, S.; Finan, C.; Swerdlow, D.I.; et al. Mendelian randomization of blood lipids for coronary heart disease. Eur. Heart J. 2015, 36, 539-550. [CrossRef]

29. Boyce, G.; Button, E.; Soo, S.; Wellington, C. The pleiotropic vasoprotective functions of high density lipoproteins (HDL). J. Biomed. Res. 2018, 32, 164-182.

30. Khalil, A.; Berrougui, H.; Pawelec, G.; Fulop, T. Impairment of the ABCA1 and SR-BI-mediated cholesterol efflux pathways and HDL anti-inflammatory activity in Alzheimer's disease. Mech. Ageing Dev. 2012, 133, 20-29. [CrossRef] [PubMed]

31. Tarasoff-Conway, J.M.; Carare, R.O.; Osorio, R.S.; Glodzik, L.; Butler, T.; Fieremans, E.; Axel, L.; Rusinek, H.; Nicholson, C.; Zlokovic, B.V.; et al. Clearance systems in the brain-implications for Alzheimer disease. Nat. Rev. Neurol. 2015, 11, 457-470. [CrossRef] [PubMed]

32. Sweeney, M.D.; Sagare, A.P.; Zlokovic, B.V. Blood-brain barrier breakdown in Alzheimer disease and other neurodegenerative disorders. Nat. Rev. Neurol. 2018, 14, 133-150. [CrossRef]

33. Hutchinson, L.; Kirk, R. High drug attrition rates-Where are we going wrong? Nat. Rev. Clin. Oncol. 2011, 8, 189-190. [CrossRef] [PubMed]

34. Sorrentino, S.A.; Besler, C.; Rohrer, L.; Meyer, M.; Heinrich, K.; Bahlmann, F.H.; Mueller, M.; Horváth, T.; Doerries, C.; Heinemann, M.; et al. Endothelial-Vasoprotective Effects of High-Density Lipoprotein Are Impaired in Patients with Type 2 Diabetes Mellitus but Are Improved after Extended-Release Niacin Therapy. Circulation 2010, 121, 110-122. [CrossRef] [PubMed]

35. Stahlman, M.; Davidsson, P.; Kanmert, I.; Rosengren, B.; Boren, J.; Fagerberg, B.; Camejo, G. Proteomics and lipids of lipoproteins isolated at low salt concentrations in D2O/sucrose or in KBr. J. Lipid Res. 2008, 49, 481-490. [CrossRef] [PubMed]

36. Hafiane, A.; Genest, J. HDL-Mediated Cellular Cholesterol Efflux Assay Method. Ann. Clin. Lab. Sci. 2015, 45, 659-668. [PubMed]

37. Gordon, S.M.; Deng, J.; Lu, L.J.; Davidson, W.S. Proteomic characterization of human plasma high density lipoprotein fractionated by gel filtration chromatography. J. Proteome Res. 2010, 9, 5239-5249. [CrossRef] [PubMed]

38. Lai, K.S.P.; Liu, C.S.; Rau, A.; Lanctôt, K.L.; Köhler, C.A.; Pakosh, M.; Carvalho, A.F.; Herrmann, N. Peripheral inflammatory markers in Alzheimer's disease: A systematic review and meta-analysis of 175 studies. J. Neurol. Neurosurg. Psychiatry 2017, 88, 876-882. [CrossRef]

39. O’Neill, F.; Riwanto, M.; Charakida, M.; Colin, S.; Manz, J.; McLoughlin, E.; Khan, T.; Klein, N.; Kay, C.W.; Patel, K.; et al. Structural and functional changes in HDL with low grade and chronic inflammation. Int. J. Cardiol. 2015, 188, 111-116. [CrossRef]

40. Ortiz-munoz, G.; Couret, D.; Lapergue, B.; Bruckert, E.; Meseguer, E.; Amarenco, P.; Meilhac, O. Dysfunctional HDL in acute stroke. Atherosclerosis 2016, 253, 75-80. [CrossRef]

41. Charakida, M.; Besler, C.; Batuca, J.R.; Wang, G. Vascular Abnormalities, Paraoxonase in Primary Antiphospholipid Syndrome. JAMA 2009, 302, 1210-1217. [CrossRef]

42. Fan, J.; Zareyan, S.; Zhao, W.; Shimizu, Y.; Pfeifer, T.A.; Tak, J.-H.; Isman, M.B.; van den Hoven, B.; Duggan, M.E.; Wood, W.; et al. Identification of a Chrysanthemic Ester as an Apolipoprotein E Inducer in Astrocytes. PLoS ONE 2016, 11, e0162384. [CrossRef] [PubMed]

43. Robert, J.; Weber, B.; Frese, L.; Emmert, M.Y.; Schmidt, D.; Von Eckardstein, A.; Rohrer, L.; Hoerstrug, S.P. A three-dimensional engineered artery model for in vitro atherosclerosis research. PLoS ONE 2013, 8, e79821. [CrossRef] [PubMed]

(c) 2019 by the authors. Licensee MDPI, Basel, Switzerland. This article is an open access article distributed under the terms and conditions of the Creative Commons Attribution (CC BY) license (http:/ / creativecommons.org/licenses/by/4.0/). 